\title{
Newer Volatile Anesthetic Agents in Cardiac Anesthesia: Review of Literature
}

\author{
Alka Mandke ${ }^{1}$ Manjula Sarkar ${ }^{2} \quad$ Charulata Deshpande ${ }^{3}$ Arun Maheshwari ${ }^{4}$ Bhupesh Kumar ${ }^{5}$ \\ M. Bhavani ${ }^{6}$ Shapna Varma ${ }^{7}$ Hetal Shah $^{8}$ Laksmi Bhanu $^{9}$ Shailesh Khanderkar ${ }^{10} \quad$ K. Ganpathy ${ }^{11}$ \\ Kunal Soni ${ }^{12}$ Reena Nebu ${ }^{13}$ Gaurav Saxena ${ }^{14}$
}

1Department of Cardiac Anaesthesia, Kokilaben Dhirubhai Ambani hospital, Mumbai, Maharashtra, India

${ }^{2}$ Department of Anaesthesia, Department of Cardiac Anaesthesia,

Dnyandeo Yashwantrao Patil Hospital, Mumbai, Maharashtra, India

Address for correspondence Gaurav Saxena, MD, Medical Division Affairs, Baxter India, 5th Floor building 9 Tower A, DLF Cyber City Phase 2,

${ }^{3}$ Department of Anaesthesia BYL, Nair Ch. Hospital, Mumbai,

Maharashtra, India

${ }^{4}$ Department of Cardiac Anaesthesia, Sir Ganga Ram hospital, New Delhi, India

${ }^{5}$ Department of Cardiac Anaesthesia, PGIMER, Chandigarh, India

6 Department of Anaesthesia, Madras Medical College, Chennai,

Tamil Nadu, India

${ }^{7}$ Department of Cardiac Anaesthesia, MGM, Healthcare, Chennai,

Tamil Nadu, India

${ }^{8}$ Department of Cardiac Anaesthesia, Narayana Hrudyalaya,

Ahmedabad, Gujrat, India

${ }^{9}$ Department of Anaesthesia, Gandhi Medical College Hyderabad,

Telangana, India

${ }^{10}$ Department of Cardiac Anaesthesia, KDA Mumbai, Maharashtra,

India

${ }^{11}$ Department of Anaesthesia, Apollo Hospitals Chennai,

Tamil Nadu, India

12Department of Anaesthesia, Apollo Hospital Bhat Ahmedabad.

Gujarat, India

${ }^{13}$ Department of Cardiac Anaesthesia, KEM Hospital Mumbai,

Maharashtra, India

${ }^{14}$ Medical Affairs Division, Baxter India, Gurgaon, Haryana, India

J Card Crit Care:2021;5:54-59

\begin{abstract}
Myocardial protection with volatile anesthetic agents have been suggested by multiple studies. These studies, however, are scattered and are often limited to a particular aspect of cardiac anesthesia. Older inhalational agents like halothane is known to cause significant hepatic damage in patients undergoing long duration surgeries while isoflurane is known to have marked vasodilating properties that also affects the coronary arteries leading to coronary "steal" phenomenon. Additionally, newer agents, like sevoflurane and desflurane, have shown more prominent cardioprotective effects than older agents. We searched ScholarOne, Medline, Embase, and the Cochrane

Keywords

- cardiac anesthesia

- inhalation anesthetic agents

- sevoflurane

- desflurane Central Register of Controlled Trials (CENTRAL) in the Cochrane Library. The medical subject headings (MeSH) terms "anaesthesia, inhalational," "anaesthesia, intravenous, or TIVA," and "Cardiac anaesthesia or Cardiac Surgery" were used. Additional studies were identified by review of the reference sections of all eligible studies. The aim of this review article is to bring together the evidences with newer inhalational agents and provide a holistic view of their benefits and shortcomings in cardiac anesthesia.
\end{abstract}

published online

March 8, 2021
Dol https://doi.org/

$10.1055 / s-0041-1723929$ ISSN 2457-0206. (c) 2021. Official Publication of The Simulation Society (TSS), accredited by International Society of Cardiovascular Ultrasound (ISCU).

This is an open access article published by Thieme under the terms of the Creative Commons Attribution-NonDerivative-NonCommercial-License, permitting copying and reproduction so long as the original work is given appropriate credit. Contents may not be used for commercial purposes, or adapted, remixed, transformed or built upon. (https://creativecommons.org/licenses/by-nc-nd/4.0/)

Thieme Medical and Scientific Publishers Pvt. Ltd. A-12, 2nd Floor, Sector 2, Noida-201301 UP, India 


\section{Introduction}

All volatile anesthetic agents show cardiac depressant effect that decrease myocardial oxygen demand and have a beneficial role on the myocardial oxygen balance during ischemia. In addition to these indirect protective effects, volatile anesthetic agents also have direct protective properties against ischemic myocardial damage. ${ }^{1}$ These properties may act as an additional tool in the treatment or prevention or both and of ischemic cardiac dysfunction in the perioperative period when implemented in clinical anesthesia. ${ }^{1}$

Indicative of cell death, an increase of cardiac biomarkers is very commonly seen in cardiac surgical procedures. Myocardial damage after cardiac surgery with significant postoperative cardiac troponin (cTn) release is associated with need for inotropic support, increased stay in intensive care unit (ICU), time on mechanical ventilation, overall hospital stay, and increased perioperative mortality rates. Number of studies have shown the potential benefits of volatile anesthetics over the intravenous agents in cardiac surgery. ${ }^{2}$ Evidence has demonstrated that patients anesthetized with volatile anesthetics tend to have lower postoperative levels of troponin as compared with intravenous anesthesia, ${ }^{3-5}$ subsequently evidence-based cardioprotective effects resulted in a decreased morbidity (including reductions of myocardial infarctions (MIs), ICU stay, duration of mechanical ventilation, overall hospital stay, and incidence of long-term cardiac events) and mortality. In a recent meta-analysis, desflurane and sevoflurane have shown to reduce postoperative mortality and the incidence of MI. ${ }^{6}$ These findings have also been extrapolated in noncardiac surgery. ${ }^{3-5}$

Underlying mechanisms of cardioprotective effects shown by volatile anesthetic agent is suggested for their ischemic preconditioning effect. ${ }^{7}$ These agents also exhibit promising postconditioning properties after cardiac surgery.

Apoptotic mRNA inhibition, cytokine/inflammation modulation, redox-sensitive pathways, neuromodulation, endothelial preservation, ion channels, and notch signaling pathways are responsible for the cardioprotective effects shown by volatile anesthetic agent at a basic level. ${ }^{8}$ These findings open a new field of investigation for potential therapies aimed to diminish secondary organ injury, as well as transplants.

\section{Unmet Needs in Cardiac Anesthesia Acute Kidney Injury}

Acute kidney injury (AKI) is a common complication following cardiac surgery. ${ }^{9,10}$ As high as $45 \%$ of patients undergoing cardiac surgery are affected by postoperative AKI and are associated with prolonged hospitalization and increased mortality. ${ }^{10-13}$ Biodegradation of volatile anesthetics into inorganic fluoride is common with both sevoflurane and enflurane. There are evidences of decreased renal concentrating ability and renal tubular injury with transient impairment and was found in patients who received sevoflurane and enflurane. ${ }^{14} \mathrm{~A}$ serum fluoride concentration of $50 \mu \mathrm{mol} / \mathrm{L}$ is the peak nephrotoxicity value.

Furthermore, the inorganic fluoride ions resulting from sevoflurane defluorination and the production of compound A with its reaction from carbon dioxide absorbent have been associated with nephrotoxicity. ${ }^{15,16}$ Fluoride levels, following the administration of isoflurane or halothane increase by 3 to 5 and 1 to $2 \mu \mathrm{mol} / \mathrm{L}$, respectively, causing the risk of nephrotoxicity to be relatively improbable.

Compare to sevoflurane or enflurane, desflurane's resistance to biodegradation allows even its prolonged exposure to be associated with normal renal function. Animal model study investigating histological tubular cell damage in a rabbit model found that desflurane preconditioning reduced renal IR injury via its protective effect on the kidneys. ${ }^{15}$

Evidences also suggest that volatile anesthetics, such as desflurane, used for anesthetic maintenance and preservation during other transplantation are associated with better outcomes than intravenous anesthetics. ${ }^{15}$ Increasing body of evidences have shown that volatile anesthetics may protect against renal ischemia reperfusion (IR) injury. ${ }^{6}$

\section{Risk of Myocardial Infarction in Cardiac Surgery}

Although experimental data have provided convincing evidence that volatile anesthetics offer protection against the consequences of reversible myocardial ischemia and reperfusion injury, the results from clinical studies in coronary surgery patients, however, have been less consistent. A more consistent result with preservation of myocardial function and a lower postoperative release of markers of myocardial damage was only found in studies where the volatile agent was administered throughout the entire surgical procedure.

Myocardial protection by volatile anesthetics during coronary surgery was evaluated by De Hert et al. Hypothesis tested in this study was that the use of a volatile agent in the anesthetic regimen would be associated with a shorter ICU and hospital length of stay (LOS), compared with a total intravenous anesthetic regimen. ${ }^{17}$

Transient troponin I increase was seen in with all anesthetic regimens used, but this increase was significantly lower with the newer volatile agent (sevoflurane and desflurane groups). ${ }^{17}$ The incidence of important myocardial damage (defined as a postoperative troponin I $>4 \mathrm{ng} / \mathrm{mL}$ ) was also significantly lower in the sevoflurane and desflurane groups. Authors hence concluded that sevoflurane and desflurane use is associated with the better preservation of early cardiac function (evident from data on hemodynamic and myocardial function, concentrations of postoperative troponin I, and need for inotropic support) and may result in an improved global tissue perfusion with a better recovery. ${ }^{17}$

\section{Perioperative Hemodynamic Stability and Ischemic Outcomes}

The anesthesiologist's traditional approach to anesthesia for coronary artery bypass graft surgery (CABG) in myocardial 
protection has emphasized the maintenance of hemodynamic stability (avoiding hypotension, hypertension, and tachycardia) and the optimization of oxygen-carrying capacity (avoiding hypoxia and anemia), thus preserving the delicate balance between myocardial oxygen supply and demand. ${ }^{17}$

In a canine model of coronary stenosis, ischemia was absent if mean aortic blood pressure exceeded heart rate, that is, if the pressure-to-rate ratio exceeded unity. ${ }^{18}$

Prevention, detection, and treatment of perioperative myocardial ischemia, regardless of the cause remains the three cornerstones of anesthetic management for patients undergoing CABG surgery. ${ }^{18}$

Numerous studies have examined the incidence of intraoperative prebypass ischemia (defined as ischemia occurring between induction of anesthesia and onset of cardiopulmonary bypass) in patients undergoing CABG surgery.

Symons and Myles, in their meta-analysis, identified 27 randomized studies including 2,979 patients comparing volatile with nonvolatile anesthesia for CABG. ${ }^{4}$ Halothane, enflurane, isoflurane, sevoflurane, and desflurane were used as volatile anaesthetic (VA) and administered during prebypass, bypass, and postbypass periods. Though there was no significant difference between volatile and nonvolatile anesthetic groups with respect to death, MI, myocardial ischemia, or ICU LOS, the patients randomized to receive volatile anesthetics had significantly higher cardiac indices, lower troponin I concentrations, shorter duration of mechanical ventilation, and shorter length of hospital stays than those randomized to receive intravenous anesthetics. Requirement for inotropic support was also reduced as compared to TIVA (Total Intra Venous Anaesthesia) group. The results suggested that volatile anesthetics may indeed be able to change outcomes in cardiac surgery. ${ }^{5}$

In another study involving 30 patients, desflurane (group $\mathrm{D}, n=10$ ), sevoflurane (group S, $n=10$ ), and isoflurane (group I, $n=10$ ) were compared for their effect on microcirculation in coronary artery bypass grafting using orthogonal polarization spectral imaging. In group $S$, the total vascular density (TVD) was decreased by $14.7 \%(p<0.05)$, the perfused vessel density (PVD) decreased by $22 \%(p<0.05)$, the proportion of perfused vessels (PPV) decreased by $5.97 \%(p<0.05)$, and the microvascular flow index (MFI) decreased by $7.69 \%(p<0.05)$. In group I, the TVD decreased by $14.7 \%$, the PVD decreased by $22 \%$, the PPV increased by $1.69 \%$, and the MFI increased by $17.99 \%(p<0.05)$. In group D, no significant changes in the TVD and PVD values were found, but the PPV increased by $1.48 \%(p<0.05)$ and the MFI increased by $8.99 \%(p<0.05)$. All these changes occurred during $\mathrm{CPB}$ and returned to baseline values 24 hours after surgery. Lactate levels did not differ statistically among the three groups. Furthermore, there were no correlations between lactate levels and these microcirculatory alterations. Authors concluded that inhalation agentsinduced transient alterations in microvascular perfusion, desflurane produced stable effects on the microcirculation. ${ }^{19}$

\section{Early Recovery after Cardiac Surgery}

Relatively higher mortality and morbidity along with higher resource utilization in cardiac surgery patients make them well suited for an enhanced recovery strategy. One of the challenges, however, in implementing uniform recommendations is the variability of institutional practice and patient characteristics. ${ }^{20}$ This was evident by the history of previous similar attempts within cardiac surgery.

Fleming et $\mathrm{al}^{21}$ compared 105 patients before and after implementing a protocol in their cardiac surgery patients similar to a colorectal early recovery after surgery (ERAS) protocol (-Table 1). The protocol included preoperative carbohydrate loading, optimizing analgesia but avoiding long-acting narcotics, nausea prophylaxis, early mobilization, and early enteral nutrition. Although they failed to demonstrate a reduced LOS, they observed improved analgesia and a reduction in a composite endpoint, including infection, AKI, respiratory failure, atrial fibrillation, MI, and death.

Sola et al outlined a protocol for enhanced recovery for transcatheter aortic valve implantation patients that provides further evidence for the feasibility of ERCS programs. ${ }^{20,21}$ (-Table 1).

\section{Fast-Track Cardiac Anesthesia}

Fast-track cardiac anesthetic techniques can lead to earlier tracheal extubation, shorter ICU stays, and significant reductions in perioperative costs and resource utilization. Whereas large-dose intravenous opioid techniques provide

Table 1 Components of ERAS for cardiac surgery (after transcatheter aortic valve implantation)

\begin{tabular}{|l|l|l|}
\hline \multicolumn{2}{|l|}{ Proposed components of Early Recovery after Cardiac Surgery ${ }^{20}$} & Postoperative \\
\hline Preoperative & Intraoperative & Multimodel analgesia \\
\hline $\begin{array}{l}\text { Patient education and well ness (preopera- } \\
\text { tive fitness, nutrition, incentive spirometry) }\end{array}$ & Short acting anesthetics & Early extubation \\
\hline Avoid prolonged fasting & Avoid high-dose, long-acting narcotics & Early mobilization \\
\hline Timely antibiotic prophylaxis & Lung protective ventilation & Incentive spirometry \\
\hline Avoid long acting sedatives & Multi model analgesia & Early tube/line removal \\
\hline Multi model analgesia & Avoid excessive volume expansion & $\begin{array}{l}\text { Aggressive PONV treatment, early oral } \\
\text { nutrition }\end{array}$ \\
\hline Anemia optimization & Timely antibiotic administration & $\begin{array}{l}\text { Delirium prevention screening and early } \\
\text { management }\end{array}$ \\
\hline
\end{tabular}

Abbreviation; PONV, post operative nausea and vomiting. 
excellent intraoperative hemodynamic stability and postoperative analgesia, extubation times are often prolonged. The use of smaller doses of opioid analgesics in combination with volatile anesthetics facilitates earlier tracheal extubation at the expense of increased postoperative pain. ${ }^{22}$

Davy et al assessed fast-track cardiac anesthesia (FTCA) in terms of the safety and resource utilization after CABG over a 1-year period. One hundred and twenty patients were initially randomized to FTCA ( $n=60$ ) or conventional anesthetic $(n=60)$ for primary elective CABG surgery.

Patients were followed for 1 year after index surgery. Acute care hospital readmission rates, LOS, and the downstream use of health resources were compared. There were no deaths during the 1-year follow-up after initial discharge; 15 (25\%) patients from both groups were readmitted to acute care hospitals during follow-up period. The mean LOS for acute care readmission was 0.3 (1.0) in the FTCA and 1.6 (6.3) days in the conventional group at 3 months; $p<0.01$, 95\% confidence interval $(\mathrm{CI})$ of $[0.1,5.7]$ and $0.8(1.8)$ and 2.9 (9.6) days at 12 months; $p<0.01,95 \% \mathrm{CI}$ of $[0.2,7.5]$. Two (3.3\%) patients in the FTCA group as compared to nine (15\%) patients in the conventional group were transferred to rehabilitation facilities. The LOS was 0.3 (1.5) and 2.3 (5.7) days, respectively; $p<0.001,95 \% \mathrm{CI}$ of $[0.6,4.0] .{ }^{23}$ Authors concluded that FTCA is a safe practice that decreases resource use for a 1-year period after index hospitalization.

Short-term results showed improvement in postextubation intrapulmonary shunt fraction and a reduction in ICU and hospital LOS..$^{23}$

\section{Volatile Anesthetic in Valvular Heart Surgeries}

Patients undergoing mitral valve surgery may exhibit postoperative depression of myocardial performance leading to postoperative morbidity and mortality. ${ }^{24}$ In MV surgery, there are prominent mechanisms of troponin release other than IR injury at work. These include cutting to remove valvular leaflets, to open the atrial wall, and "acute afterload mismatch" because of partial or total destruction of the subvalvular apparatus. These would all result in significant increase in end systolic circumferential wall stress and geometric distortion of the left ventricle. The choice of an anesthetic that preserves myocardial function may help prevent such postoperative cardiac dysfunction..$^{24,25}$ In 120 patients who were undergoing СРB for mitral valve surgery, a subgroup analysis of 20 patients with concomitant coronary artery disease showed reduction $(p<0.02)$ of median peak of troponin I in patients receiving desflurane compared with patients receiving TIVA (14.0 vs. $31.6 \mathrm{ng} / \mathrm{dL}$ ). In same subgroup, seven of eight patients receiving desflurane underwent CABG surgery, whereas 9 of 12 patients receiving propofol underwent CABG surgery. Notably, no patient died among the eight patients who received desflurane, whereas 2 of 11 (18\%) deaths were observed among the patients who received TIVA for MV surgery with concomitant CABG surgery. ${ }^{25}$

In 76 patients, New York Heart Association (NYHA) classes II to III, presenting electively for aortic valve regurgitation (AVR) for severe symptomatic aortic stenosis (AS) and randomized either into desflurane or TIVA group, desflurane resulted in shorter duration of postoperative mechanical ventilation, ICU and hospital stays, as well as cTn I (cTnI). There was no difference in terms of 30-day mortality, incidence of arrhythmia, or need for cardioversion between the two groups (- Table 2). IMA has gained acceptance as marker for evaluation of myocardial injuries. Though no significant difference was found in levels of ischemia-modified albumin (IMA) and cTnI between study groups, post-CPB cTnI level was significantly higher than baseline in the TIVA group, showing a cardioprotective ischemia preconditioning effect of desflurane. TIVA group had lower mean arterial pressure (MAP) and recorded lower inotrope use. ${ }^{26}$

\section{Cardiac Patients in Noncardiac Surgeries}

Patients who undergo noncardiac surgery after coronary stent insertion are at an increased risk of perioperative cardiovascular complications.

In a study of 1,630 patients done to understand the association between anesthetic agents and the risk of major adverse cardiovascular and cerebral event (MACCE) in patients undergoing noncardiac surgery with history of stent implantation 975 patients (59.8\%) received TIVA, and 655 patients received volatile anesthetic (40.2\% including sevoflurane $26.9 \%$ and desflurane $13.3 \%$ ) during the surgery. ${ }^{26}$ Unmatched incidence of MACCE was significantly higher in the sevoflurane group than in the TIVA group (sevoflurane $8.2 \%$ vs. TIVA 5.1\%, $p=0.006$ ). However, there was no significant difference between the volatile and the TIVA groups (volatile group $7.2 \%$ vs. TIVA $5.1 \%, p=0.087$ ). On comparison of individual components of MACCE in respective groups, the incidence of pulmonary embolism was significantly higher in sevoflurane group compared to TIVA group (sevoflurane $1.6 \%$ vs. TIVA $0.2 \%, p=0.005$ ). Incidence of MACCE groups of any pair (TIVA 5.6 vs. volatile anesthetics $6.9 \%, p=0.356$; and TIVA $6.5 \%$ vs. sevoflurane $7.7 \%, p=0.507)^{27}$ was not significantly different after the propensity score matching.

The choice of anesthetic agent for noncardiac surgery did not significantly affect the development of MACCE in patients with previous coronary stent implantation. However, further

Table 2 Comparison of the effects of inhalational anesthesia with desflurane and total intravenous anesthesia on cardiac biomarkers after aortic valve replacement

\begin{tabular}{|l|l|l|l|}
\hline Parameters & TIVA & Desflurane & $p$-Value \\
\hline $\begin{array}{l}\text { Duration of mechanical } \\
\text { ventilation }\end{array}$ & 14.03 & 9 & 0.000 \\
\hline ICU stays days & 4.28 & 3.5 & 0.003 \\
\hline Hospital stays days & 7.92 & 7.02 & 0.009 \\
\hline $\begin{array}{l}\text { Duration of inotropes } \\
\text { hours }\end{array}$ & 52.86 & 62.08 & 0.000 \\
\hline Incidence of SVT & $16.70 \%$ & $17.50 \%$ & 0.923 \\
\hline Incidence of VT/VF & $19.40 \%$ & $17.50 \%$ & 0.827 \\
\hline 30-day mortality & 2 & 3 & 0.733 \\
\hline
\end{tabular}

Abbreviation: ICU, intensive care unit; SVT, supra ventricular tachycardia; TIVA, total intra venous anaesthesia; VF, ventricular fibrillation; VT, ventricular tachycardia. 
randomized trials are needed to confirm the results as in case of cardiac surgical patients with a high risk of postoperative myocardial injury, previous meta-analyses have reported reduced mortality after cardiac surgery when volatile anesthetics were used compared to TIVA. ${ }^{28}$

In another recent meta-analysis including 68 randomized trials, general anesthesia with volatile anesthetics was found to be associated with reduced mortality and a lower incidence of postoperative pulmonary complications following cardiac surgery. ${ }^{28}$

Several meta-analyses have shown that desflurane and sevoflurane could reduce postoperative mortality and incidence of MI following cardiac surgery with significant advantages in terms of reduced postoperative cTnI release, need for inotropic support, time on mechanical ventilation, ICU, and overall hospital stay. Decreased myocardial oxygen demand with volatile anesthetic will require further exploratory studies in this regard by a well-planned randomized controlled trial. Desflurane owing to its hemodynamic stability minimal cardiovascular depression and low solubility (lower partition coefficient) may offer better outcomes in variety of cardiac surgeries. The study in 60 elective patients with left ventricular ejection fraction above $30 \%$ indicated that desflurane might accelerate recovery of myocardial contractility with improved stability. ${ }^{29}$

\section{Conclusion}

With FTCA gaining popularity globally and eventually in country, it can portray desflurane as a unique new volatile anesthetic agent with significantly quicker recovery and better hemodynamic stability in patients undergoing cardiac surgery and cardiac patients undergoing noncardiac surgeries.

\section{Source(s) of Support}

None.

\section{Conflict of Interest}

Corresponding author G.S. is on pay roll of Baxter India Pvt. Ltd. which is the innovator of all inhalational agents.

\section{References}

1 Landoni G, Fochi O, Torri G. Cardiac protection by volatile anaesthetics: a review. Curr Vasc Pharmacol 2008;6(2):108-111

2 Straarup TS, Hausenloy DJ, Rolighed Larsen JK. Cardiac troponins and volatile anaesthetics in coronary artery bypass graft surgery: a systematic review, meta-analysis and trial sequential analysis. Eur J Anaesthesiol 2016;33(6):396-407

3 Marret E, Piriou V. [Should volatile anaesthetics decrease morbidity and mortality after cardiac surgery? Comments of three recent meta-analysis] (in French) Ann Fr Anesth Reanim 2008;27(3):280-283

4 Symons JA, Myles PS. Myocardial protection with volatile anaesthetic agents during coronary artery bypass surgery: a meta-analysis. Br J Anaesth 2006;97(2):127-136

$5 \mathrm{Yu} \mathrm{CH}$, Beattie WS. The effects of volatile anesthetics on cardiac ischemic complications and mortality in CABG: a meta-analysis. Can J Anaesth 2006;53(9):906-918

6 Landoni G, Biondi-Zoccai GG, Zangrillo A, et al. Desflurane and sevoflurane in cardiac surgery: a meta-analysis of randomized clinical trials. J Cardiothorac Vasc Anesth 2007;21(4):502-511
7 Landoni G, Bignami E, Oliviero F, Zangrillo A. Halogenated anaesthetics and cardiac protection in cardiac and non-cardiac anaesthesia. Ann Card Anaesth 2009;12(1):4-9

8 Fleisher LA, Beckman JA, Brown KA, et al. ACC/AHA 2007 guidelines on perioperative cardiovascular evaluation and care for noncardiac surgery: executive summary: a report of the American College of Cardiology/American Heart Association Task Force on Practice Guidelines (writing committee to revise the 2002 guidelines on perioperative cardiovascular evaluation for noncardiac surgery) developed in collaboration With the American Society of Echocardiography, American Society of Nuclear Cardiology, Heart Rhythm Society, Society of Cardiovascular Anesthesiologists, Society for Cardiovascular Angiography and Interventions, Society for Vascular Medicine and Biology, and Society for Vascular Surgery. J Am Coll Cardiol 2007;50(17):1707-1732

9 Rosner MH, Okusa MD. Acute kidney injury associated with cardiac surgery. Clin J Am Soc Nephrol 2006;1(1):19-32

10 Shaw A. Update on acute kidney injury after cardiac surgery. J Thorac Cardiovasc Surg 2012;143(3):676-681

11 Koyner JL, Bennett MR, Worcester EM, et al. Urinary cystatin $\mathrm{C}$ as an early biomarker of acute kidney injury following adult cardiothoracic surgery. Kidney Int 2008;74(8):1059-1069

12 Fang Y, Ding X, Zhong Y, et al. Acute kidney injury in a Chinese hospitalized population. Blood Purif 2010;30(2):120-126

13 Mitter N, Shah A, Yuh D, et al. Renal injury is associated with operative mortality after cardiac surgery for women and men. J Thorac Cardiovasc Surg 2010;140(6):1367-1373

14 Gentz BA, Malan TP Jr. Renal toxicity with sevoflurane: a storm in a teacup? Drugs 2001;61(15):2155-2162

15 Motayagheni N, Phan S, Eshraghi C, Nozari A, Atala A. A review of anesthetic effects on renal function: potential organ protection. Am J Nephrol 2017;46(5):380-389

16 Kobayashi Y, Ochiai R, Takeda J, Sekiguchi H, Fukushima K. Serum and urinary inorganic fluoride concentrations after prolonged inhalation of sevoflurane in humans. Anesth Analg 1992;74(5):753-757

17 De Hert SG, Van der Linden PJ, Cromheecke S, et al. Choice of primary anesthetic regimen can influence intensive care unit length of stay after coronary surgery with cardiopulmonary bypass. Anesthesiology 2004;101(1):9-20

18 Lonjaret L, Lairez O, Minville V, Geeraerts T. Optimal perioperative management of arterial blood pressure. Integr Blood Press Control 2014;7:49-59

19 Özarslan NG, Ayhan B, Kanbak M, et al. Comparison of the effects of sevoflurane, isoflurane, and desflurane on microcirculation in coronary artery bypass graft surgery. J Cardiothorac Vasc Anesth 2012;26(5):791-798

20 Sola M, Ramm CJ, Kolarczyk LM, et al. Application of a multidisciplinary enhanced recovery after surgery pathway to improve patient outcomes after transcatheter aortic valve implantation. Am J Cardiol 2016;118(3):418-423

21 Fleming IO, Garratt C, Guha R, et al. Aggregation of marginal gains in cardiac surgery: feasibility of a perioperative care bundle for enhanced recovery in cardiac surgical patients. J Cardiothorac Vasc Anesth 2016;30(3):665-670

22 Zarate E, Latham P, White PF, et al. Fast-track cardiac anesthesia: use of remifentanil combined with intrathecal morphine as an alternative to sufentanil during desflurane anesthesia. Anesth Analg 2000;91(2):283-287

23 Cheng DC, Wall C, Djaiani G, et al. Randomized assessment of resource use in fast-track cardiac surgery 1-year after hospital discharge. Anesthesiology 2003;98(3):651-657

24 Landoni G, Calabrò MG, Marchetti C, et al. Desflurane versus propofol in patients undergoing mitral valve surgery. J Cardiothorac Vasc Anesth 2007;21(5):672-677

25 Martin AK, Malhotra AK, Sullivan BL, Ramakrishna H. Troponin elevations in patients with chronic cardiovascular disease: 
an analysis of current evidence and significance. Ann Card Anaesth 2016;19(2):321-327

26 Kapoor PM, Taneja S, Kiran U, Rajashekhar P. Comparison of the effects of inhalational anesthesia with desflurane and total intravenous anesthesia on cardiac biomarkers after aortic valve replacement. Ann Card Anaesth 2015;18(4):502-509

27 Yoon HK, Jun K, Park SK, et al. Anesthetic agents and cardiovascular outcomes of noncardiac surgery after coronary stent insertion. J Clin Med 2020;9(2):429
28 Uhlig C, Bluth T, Schwarz K, et al. Effects of volatile anesthetics on mortality and postoperative pulmonary and other complications in patients undergoing surgery: a systematic review and meta-analysis. Anesthesiology 2016;124(6):1230-1245

29 Mroziński P, Lango R, Biedrzycka A, Kowalik MM, Pawlaczyk $\mathrm{R}$, Rogowski J. Comparison of haemodynamics and myocardial injury markers under desflurane vs. propofol anaesthesia for off-pump coronary surgery. A prospective randomised trial. Anaesthesiol Intensive Ther 2014;46(1):4-13 